Differential effect of cigarette smoke exposure on exhaled nitric oxide and blood eosinophils in healthy and asthmatic individuals

This content has been downloaded from IOPscience. Please scroll down to see the full text.

2017 J. Breath Res. 11036006

(http://iopscience.iop.org/1752-7163/11/3/036006)

View the table of contents for this issue, or go to the journal homepage for more

Download details:

IP Address: 128.83.63.20

This content was downloaded on $26 / 08 / 2017$ at $14: 20$

Please note that terms and conditions apply.

You may also be interested in:

Evolution of exhaled nitric oxide levels throughout development and aging of healthy humans

Tiago Jacinto, Andrei Malinovschi, Christer Janson et al.

Biomarkers in exhaled breath condensate

N M Grob, M Aytekin and R A Dweik

Exhaled nitric oxide measurements in patients with acute-onset interstitial lung disease

Keiji Oishi, Tsunahiko Hirano, Ryo Suetake et al.

Nasal nitric oxide is associated with exhaled NO, bronchial responsiveness and poor asthma control

C Krantz, C Janson, M P Borres et al.

Exhaled nitric oxide in a middle-aged Icelandic population cohort

Anna Kristin Thorhallsdottir, David Gislason, Andrei Malinovschi et al.

Exhaled and non-exhaled non-invasive markers for assessment of respiratory inflammation in patients with stable COPD and healthy smokers

Giuseppe Santini, Nadia Mores, Rugia Shohreh et al.

Exhaled carbon monoxide in lung disease

Stefan W Ryter and Jigme M Sethi

Ultrafine particle content in exhaled breath condensate in airways of asthmatic children

Shira Benor, Yfat Alcalay, Keren Armoni Domany et al. 


\title{
Differential effect of cigarette smoke exposure on exhaled nitric oxide and blood eosinophils in healthy and asthmatic individuals
}

\author{
Tiago Jacinto ${ }^{1,2,3,8}$, Andrei Malinovschi ${ }^{4}$, Christer Janson ${ }^{5}$, João Fonseca ${ }^{1,2,6}$ and Kjell Alving \\ 1 Department of Allergy: Instituto \& Hospital CUF, Porto, Portugal \\ 2 CINTESIS- Center for Health Technology and Services Research, Faculty of Medicine, University of Porto, Portugal \\ Department of Cardiovascular and Respiratory Sciences, Porto Health School, Porto, Portugal \\ 4 Department of Medical Sciences: Clinical Physiology, Uppsala University, Uppsala, Sweden \\ 5 Department of Medical Sciences: Respiratory, Allergy and Sleep Research, Uppsala University, Uppsala, Sweden \\ 6 MEDCIDS- Department of Community Medicine, Information, and Health Sciences: Faculty of Medicine, University of Porto, Portugal \\ Department of Women's and Children's Health: Paediatric Research, Uppsala University, Uppsala, Sweden \\ 8 Author to whom any correspondence should be addressed. \\ E-mail: tajacinto@gmail.com, andrei.malinovschi@medsci.uu.se, christer.janson@medsci.uu.se,fonseca.ja@gmail.com andkjell. \\ alving@kbh.uu.se
}

Keywords: exhaled nitric oxide, blood eosinophils, smoking, asthma, inflammation

\begin{abstract}
Background: Tobacco smoking affects both the fraction of exhaled nitric oxide (FeNO) and blood eosinophil (B-Eos) count, two clinically useful biomarkers in respiratory disease that represent local and systemic type-2 inflammation, respectively. Objective: We aimed to study the influence of objectively measured smoke exposure on FeNO and B-Eos in a large population of subjects with and without asthma. Methods: We utilized the US National Health and Nutrition Examination Surveys 2007-2012 and included 10669 subjects aged 6-80 years: 9869 controls and 800 asthmatics. Controls were defined as having no respiratory disease, no hay fever in the past year, and B-Eos count $\leqslant 0.3 \times$ $10^{9} 1^{-1}$. Asthma was defined as self-reported current asthma and at least one episode of wheezing or an asthma attack in the past year, but no emphysema or chronic bronchitis. Tobacco use was collected via questionnaires and serum cotinine was measured with mass spectrometry. Results: Increasing cotinine levels were associated with a progressive reduction in FeNO in both controls and asthmatics. FeNO remained significantly higher in asthmatics than controls except in the highest cotinine decile, equivalent to an average reported consumption of 13 cigarettes/day. B-Eos count increased with cotinine in controls, but was unchanging in asthmatics. Interestingly, B-Eos count was significantly higher in presently non-exposed (cotinine below detection limit) former smokers than never smokers. Conclusion: Smoke exposure decreases FeNO and increases B-Eos count. These effects should be considered in the development of normalized values and their interpretation in clinical practice. The persistence of elevated B-Eos in former smokers warrants further studies.
\end{abstract}

\section{Introduction}

Asthma is a chronic disease, characterized by inflammation of the airways, and defined by respiratory symptoms, such as wheeze and shortness of breath, along with variable expiratory airflow limitation [1]. Various phenotypes are described, such as atopic asthma with eosinophilia, non-atopic eosinophilic asthma, and type2-low asthma [2]. These manifestations of the disease can be evaluated using biomarkers such as the fraction of exhaled nitric oxide (FeNO) and blood eosinophil (B-Eos) count [3]. FeNO is recognized as a marker of airway inflammation that is primarily triggered by IL-4 and IL-13 [4], and is increasingly used in clinical practice [5]. Elevated B-Eos is a marker of systemic eosinophilic inflammation, common in asthma [6], and is primarily triggered by IL- 5 [4]. It has been shown that it might be advantageous to assess both these pathways of inflammation, as both local and systemic type- 2 inflammation, independent of each other, are important for the 


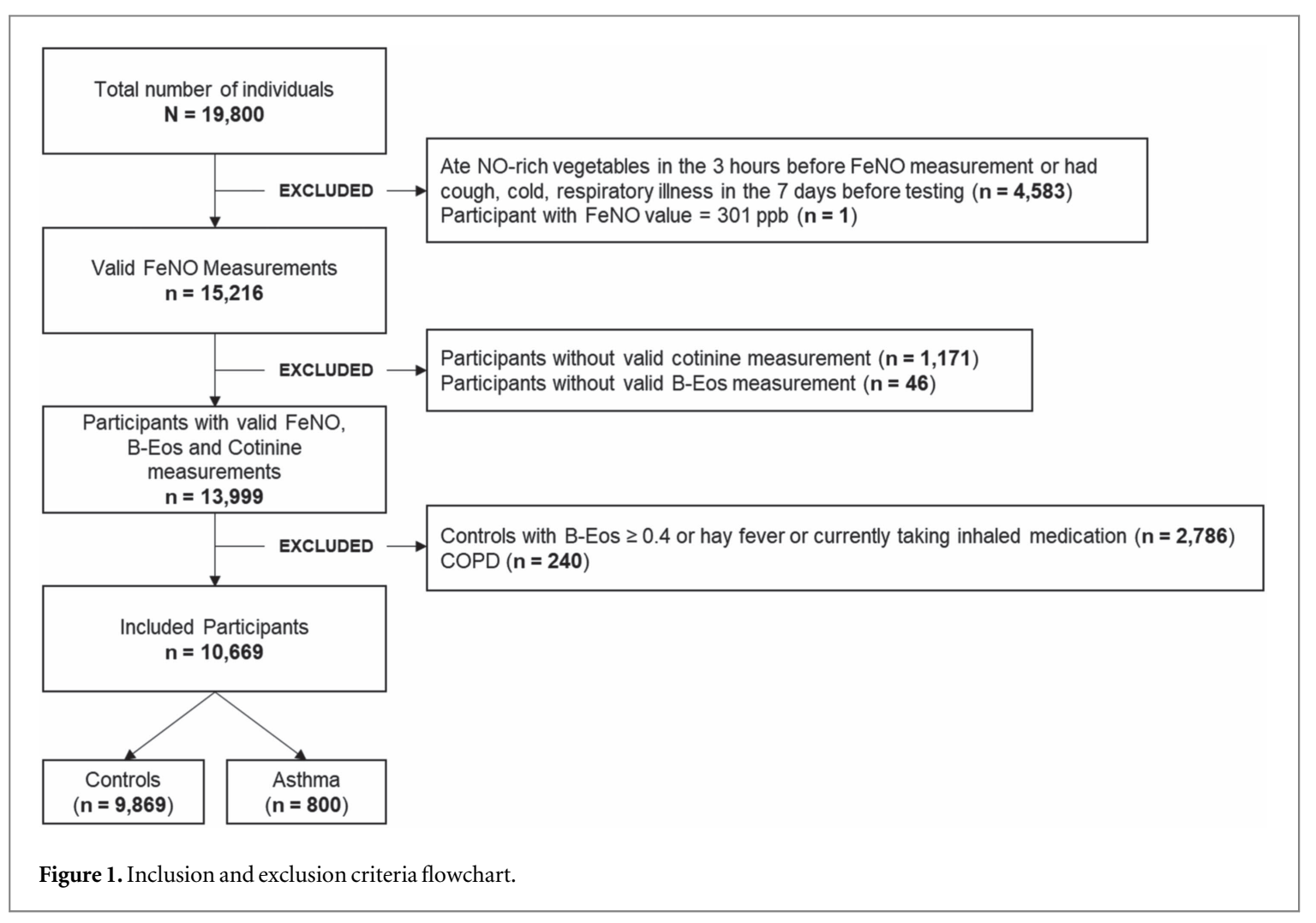

development of respiratory symptoms and clinical asthma [3, 7].

However, FeNO and B-Eos count are both affected by endogenous and exogenous factors [8, 9], For example, both current and past smoking were associated with reduced airway and alveolar levels of NO [10]. In contrast, smokers were shown to have increased numbers of eosinophils in induced sputum samples [11].

One of the limitations of determining smoking habits and exposure to tobacco smoke is the use of self-reported measures, which seem to have a poor correlation with objective markers such as cotinine, a metabolite of nicotine [12]. Further, cotinine measurement seems to be especially useful to correctly determine the amount of passive smoking [13]. Cotinine measurement in blood seems to have better performance than measurements in urine or saliva [14].

Thus, there is evidence of inflammatory changes caused by smoking in asthmatics, changes that could lead to a decreased response to corticosteroids and increased susceptibility to infection [15]. Moreover, increased serum cotinine was found to be associated with an increased number of asthma exacerbations and healthcare usage in non-smokers with asthma [16].

In this study, we aim to investigate the influence of active and passive exposure to cigarette smoke, objectively determined using serum cotinine, on FeNO and B-Eos count, in (a) healthy children and adults with no history of respiratory diseases, and (b) individuals with self-reported asthma, who have been included in the

National Health and Nutrition Examination Surveys 2007-2012.

\section{Methods}

\section{Participants}

The participants of this study were selected from the publicly available datasets of the National Health and Nutrition Examination Survey (NHANES), which is a nationally representative series of studies designed to assess the health and nutritional status of adults and children in the United States, combining interviews and physical examinations conducted by the National Centre for Health Statistics, a part of the Centre for Disease Control and Prevention. More detailed information is available online at: www.cdc.gov/nchs/ nhanes/about_nhanes.htm.

From a total of 30442 participants in NHANES 2007-2012, we have included individuals that performed a FeNO measurement $(n=19800 ; 65 \%)$. Of these, we have excluded 4583 individuals who ate NOrich vegetables in the $3 \mathrm{~h}$ before FeNO measurement, or had a cough, cold, or respiratory illness in the seven days before testing, and one individual with a FeNO value of $>300 \mathrm{ppb}$. Furthermore, we have excluded participants without a valid cotinine $(n=1171)$ or B-Eos $(n=46)$ measurement. A detailed flowchart with the inclusion and exclusion criteria is shown in figure 1. 
Two groups were created, (1) controls $(n=9869)$ and (2) asthma $(n=800)$. Healthy individuals were defined as having no previous diagnosis of asthma, emphysema, or chronic bronchitis, and no hay fever in the past 12 months. Participants with B-Eos $\geqslant 0.4$ or reporting hay fever in the past 12 months or currently taking inhaled medication were excluded $(n=2786)$, as were those reporting having COPD $(n=240)$. Asthma was defined as self-reported physiciandiagnosed asthma at any time of life together with a positive answer to the following question: 'Do you still have asthma?' and at least one episode of wheezing or an asthma attack in the past 12 months.

\section{Exhaled NO}

FeNO was measured before spirometry was performed with an electrochemical analyser (NIOX MINO; Aerocrine $A B$, Solna, Sweden) in subjects aged $\geqslant 6$ years. A valid measurement was defined in accordance with ATS/ERS guidelines [17] as: having two reproducible measurements at an expiratory flow rate of $50 \mathrm{ml} \mathrm{s}^{-1}$, no use of oral or inhaled steroids in the past two days, no breathing problems requiring oxygen, no problems taking deep breaths, no strenuous exercise in the hour prior to the measurement, in addition to the exclusion criteria described above. Up to four FeNO measurement attempts were made and the mean of two reproducible FeNO measurements (within $2 \mathrm{ppb}$ if levels were $<30 \mathrm{ppb}$ or within $10 \%$ if levels were $>30 \mathrm{ppb}$ ) was taken as the final result. The lower and upper detection limits of NIOX MINO are 5 and $300 \mathrm{ppb}$, respectively. If two measurements were below the limit of detection of the device ( $5 \mathrm{ppb}$ ), a value of $3.5 \mathrm{ppb}$ (lower limit of detection divided by the square root of two) was used as the mean.

\section{Blood eosinophils}

Blood was collected from participants by a phlebotomist. Complete blood count was analysed using the Coulter method. The white blood cell differential uses VCS technology. Analysis and classification of WBCs use three simultaneous measurements of individual cell volume $(V)$, high frequency conductivity $(C)$, and laser light scatter $(S)$. The parameters analysed were the absolute and relative number of eosinophils. Further details are available (https://n.cdc.gov/ Nchs/Nhanes/2011-2012/CBC_G.htm).

\section{Smoking habits}

Included individuals were categorized into three groups: current smokers, former smokers, and never smokers. Current smokers were defined as subjects who reported smoking at least 100 cigarettes in their lifetime and who, at the time of survey, smoked either every day or some days. Former smokers were defined as subjects who reported smoking at least 100 cigarettes in their lifetime and who, at the time of the survey, did not smoke at all. Never smokers were defined as individuals who reported having smoked less than 100 cigarettes in their lifetime. An additional category was created, non-smokers, by adding the former smokers to the never smokers, representing the individuals that did not currently smoke, regardless of past smoking habits. Since the questions regarding smoking habits were only posed to adults, the included individuals were divided into two groups: children (age $<18$ years) and adults (age $\geqslant 18$ years).

\section{Cotinine}

Cotinine is a major metabolite of nicotine that can be used as a marker for active smoking and as an index for environmental tobacco smoke exposure. Cotinine is generally preferred over nicotine for such assessments because of its substantially longer half-life [18, 19]. Cotinine was measured in the participant's serum, using an isotope-dilution high-performance liquid chromatography/atmospheric pressure chemical ionization tandem mass spectrometric (ID HPLCAPCI MS/MS) method. The detection limit was $0.015 \mathrm{ng} \mathrm{ml}^{-1}$. If the participant had a cotinine value below the detection limit, the detection limit divided by the square root of two was used. Further details are available (https://n.cdc.gov/Nchs/Nhanes/20112012/COTNAL_G.htm).

The number of individuals consuming other forms of tobacco or under nicotine replacement therapy was very low in both the control group (chewing tobacco- $n=106,1.1 \%$; snuff- $n=44,0.4 \%$; patches, gums, others $-n=24,0.2 \%)$ and the asthma group (chewing tobacco- $n=3,0.4 \%$; snuff $-n=1$, $0.1 \%$; patches, gums, other $-n=2,0.2 \%$ ), and did not have a significant impact on the results (data not shown). There was no information available on the usage of e-cigarettes, although we believe that this usage would be minimal during the survey years 2007-2012.

\section{Ethics}

All protocols were approved by the Ethics Review Board of the National Centre for Health Statistics Research. All participants provided written informed consent.

\section{Statistical analysis}

The skewness of the distribution of continuous variables was assessed with the Kolmogorov-Smirnov test and visual assessment of the histograms. The reference group was composed of participants with cotinine levels equal to or less than the detection limit of $0.015 \mathrm{ng} \mathrm{ml}^{-1}$ ( $n=2610,24.5 \%$ ). The remaining groups were created using percentiles 10,50 and 90 in all participants (controls and asthmatics together) with a cotinine value greater than the lower limit of detection; these percentiles were chosen to better accommodate the skewed distribution of cotinine and to allow incremental average numbers of cigarettes 
Table 1. Comparison of the average number of cigarettes smoked in the past 30 days between different cotinine strata.

\begin{tabular}{lrr}
\hline Cotinine strata & $n$ & $\begin{array}{l}\text { Average number of cigarettes } \\
\text { smoked in the past 30 days }\end{array}$ \\
\hline $\begin{array}{l}\text { Percentiles 10, 50 } \\
\text { and 90 }\end{array}$ & \\
\hline $0.016-0.021$ & 655 & 1.6 \\
$0.022-0.164$ & 2388 & 2.3 \\
$0.165-283.0$ & 2376 & 6.5 \\
$>283.0$ & 539 & 12.7 \\
\hline Percentiles 25, 50 & & \\
$\quad$ and 75 & & \\
\hline $0.016-0.034$ & 1519 & 2.5 \\
$0.035-0.164$ & 1524 & 2.1 \\
$0.165-86.7$ & 1519 & 3.3 \\
$>86.7$ & 1426 & 10.3 \\
\hline
\end{tabular}

smoked per day (geometric mean) in the different strata of serum cotinine as shown in table 1 .

To obtain normal distributions, FeNO and B-Eos were natural log-transformed before the analysis, and described using geometric means and 95\% confidence intervals. Both these variables were corrected for age and gender, when applicable [20]. Associations were tested with one-way and two-way ANOVA tests, when applicable, with $\ln (\mathrm{FeNO})$ and $\ln$ (B-Eos) as the dependent variable, which was back-transformed for interpretation. Relative differences in the geometric mean between the reference stratum and the other strata were calculated using the formula: (Stratum-Reference Group)/ Reference Group * 100. All the statistical analyses were performed using IBM SPSS Statistics 24 (Chicago, IL, USA) and Graphpad Prism v 6.05 (La Jolla, CA, USA). The significance level was set to $\alpha=0.05$.

\section{Results}

We have included 10669 subjects (54\% of total NHANES participants aged six years and up during the survey years 2007-2012) with valid FeNO, B-Eos, and cotinine measurements: 9869 (92.5\%) healthy controls and $800(7.5 \%)$ participants with asthma (figure 1). Participant characteristics are shown in table 2 .

There were slightly more females in the asthma group than in the control group, and the controls had a higher mean age than the asthma group. No significant effect of medication on FeNO and B-Eos count in the different cotinine strata was found. There were more smokers in the asthma group, and more never smokers in the control group, whereas the proportion of former smokers was similar among asthmatics and controls. In addition, a proportion of 7.6\% of former smokers and $7.5 \%$ of never smokers presented with high levels of cotinine when analysing controls and asthmatics together (figure E1 is available
Table 2. Description of included individuals.

\begin{tabular}{|c|c|c|c|}
\hline & $\begin{array}{l}\text { Controls } \\
n=9869\end{array}$ & $\begin{array}{l}\text { Asthma } \\
n=800\end{array}$ & $p$ \\
\hline \multicolumn{4}{|l|}{ Gender } \\
\hline Male $n(\%)$ & $5012(51)$ & $374(47)$ & 0.028 \\
\hline Female $n(\%)$ & 4857 (49) & $426(53)$ & \\
\hline Age (years) & $35.8(20.5)$ & $29.7(19.6)$ & $<0.001$ \\
\hline Age $(<18$ years $) n(\%)$ & $2507(25)$ & $306(38)$ & $<0.001$ \\
\hline Age $(\geqslant 18$ years $) n(\%)$ & $7362(75)$ & $494(62)$ & \\
\hline \multicolumn{4}{|l|}{ Smoking status $n(\%)$} \\
\hline Current smoker & $1385(20.0)$ & $125(27.7)$ & $<0.001$ \\
\hline Former smoker & $1534(22.2)$ & $106(23.5)$ & NS \\
\hline Never smoker & $3996(57.8)$ & $221(48.9)$ & $<0.001$ \\
\hline \multicolumn{4}{|l|}{ Medication use $n(\%) \#$} \\
\hline $\begin{array}{l}\text { Inhaled or oral } \\
\text { corticosteroids }\end{array}$ & NA & $168(21)$ & \\
\hline $\begin{array}{l}\text { Leukotriene } \\
\text { modifiers }\end{array}$ & NA & $80(10)$ & \\
\hline \multicolumn{4}{|l|}{ Cotinine $n(\%)$} \\
\hline$\leqslant 0.015$ & $2453(25)$ & $157(20)$ & 0.001 \\
\hline $0.016-0.021$ & $867(9)$ & $62(8)$ & \\
\hline $0.022-0.122$ & $2935(30)$ & $235(29)$ & \\
\hline $0.123-239.6$ & $2903(29)$ & $282(35)$ & \\
\hline$>239.6$ & $711(7)$ & $62(8)$ & \\
\hline $\mathrm{BMI}\left(\mathrm{kg} \mathrm{m}^{-2}\right)$ & $26.5(7.0)$ & $27.2(8.5)$ & 0.009 \\
\hline $\begin{array}{l}\text { Blood eosinophils } \\
\qquad\left(1000 \text { cells } \mu \mathrm{l}^{-1}\right)\end{array}$ & $127(0.7)$ & $212(8.2)$ & $<0.001$ \\
\hline FENO (ppb) & $11.9(0.1)$ & $15.9(0.9)$ & $<0.001$ \\
\hline
\end{tabular}

All categorical variables are represented by $n(\%)$. Age and body mass index (BMI) are represented by mean (SD). The remaining variables are represented by geometric mean (SEM). NS: not significant. NA: not available. \#: Participants were asked to refrain from taking medication at least 2 days before FeNO measurement.

online at stacks.iop.org/JBR/11/036006/mmedia). The asthma group had higher B-Eos count, FeNO and cotinine levels ( 0.301 versus $0.207 \mathrm{ng} \mathrm{ml}^{-1}, p<0.001$ ) compared with the control group. The proportions of the different cotinine groups were mostly equally distributed, with the exception of the reference stratum, with a higher proportion in the control group, and the second highest stratum, with a higher proportion in the asthma group.

Increasing cotinine levels were associated with lower levels of FeNO (figure 2(A)). There were significant differences compared with the reference stratum for the two highest cotinine strata, corresponding to an average of 6.5 or 12.7 cigarettes smoked per day, respectively. Moreover, in the highest cotinine stratum, the difference in FeNO between controls and asthmatics had disappeared (table 3 ). The relative differences were gradual, with a decrease of $42 \%$ in the control group and $64 \%$ in the asthma group in the 


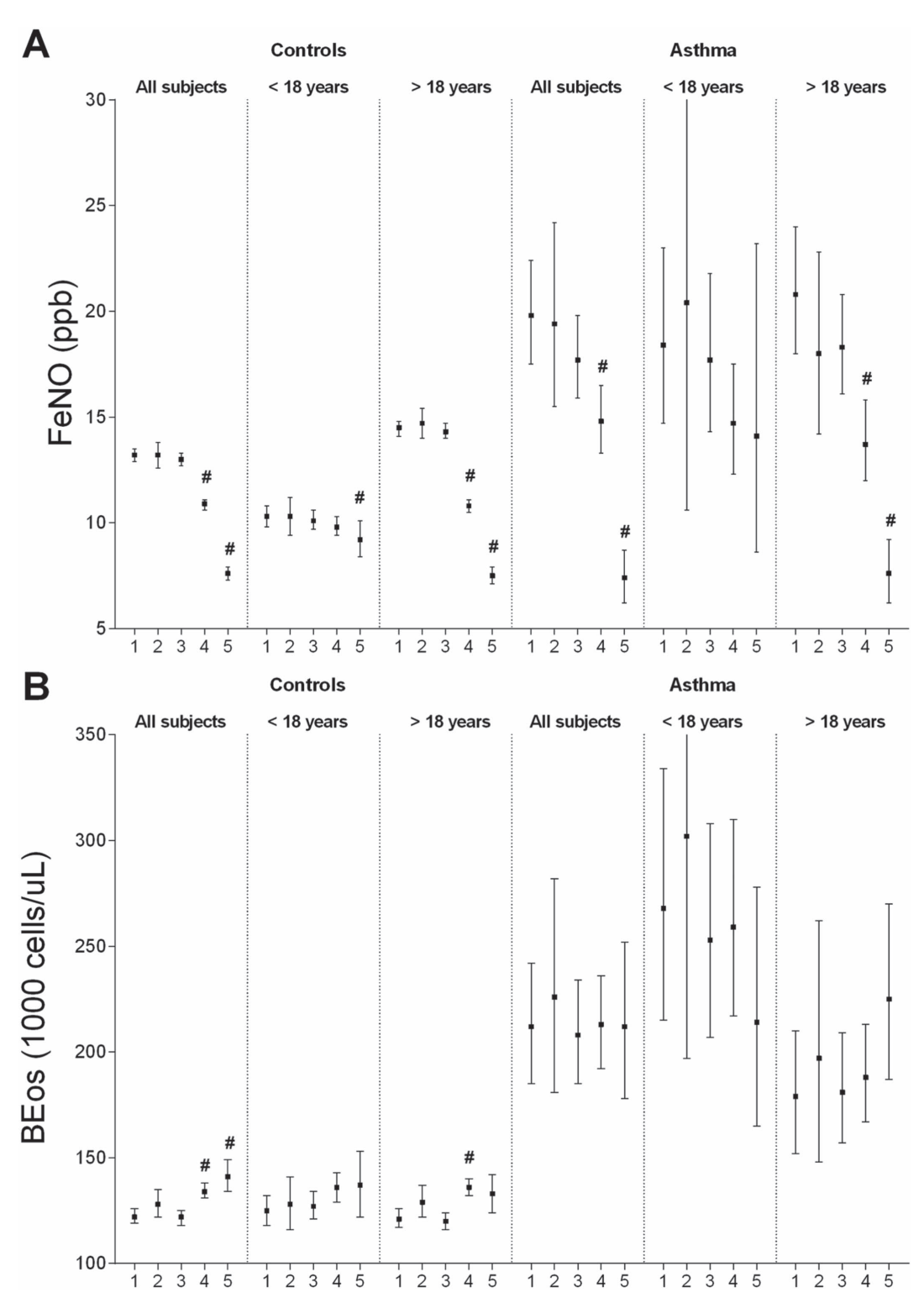

Figure 2. Relative differences of FeNO and B-Eos between cotinine strata and the reference stratum, in controls and asthma. FeNO and B-Eos are represented by geometric mean and $95 \%$ confidence intervals (filled squares and circle with whiskers, respectively). \#: Significant difference from the reference cotinine stratum $(\leqslant 0.015)$ at $p<0.001$ (corrected for age). Note that the cotinine strata are not the same in the different groups; categories: (a) all subjects: 1 ' $\leqslant 0.015$ ’, $2^{`} 0.016-0.021^{\prime}, 3^{\prime} 0.022-0.122^{\prime}, 4^{`} 0.123-239.6^{\prime}, 5$

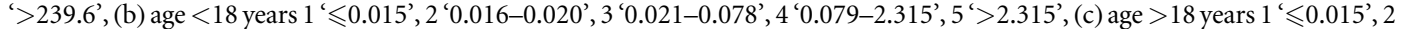
'0.016-0.021', 3 ' $0.022-0.164$ ', 4 ' $0.165-283.0$ ', 5'>283.0'.

highest cotinine stratum. The absolute difference was larger in asthmatics than controls for most cotinine strata, at least in adults (table E1).

The cotinine strata, corresponding to the percentiles 10, 50 and 90 of each age group, differed between children and adults, which should be recognized when interpreting the results.

A contrasting effect of tobacco smoke exposure was seen for the B-Eos count, as shown in figure 2(B). There was no clear effect of increasing cotinine levels in asthmatics, but there was a significant effect in controls, with increasing B-Eos counts in the two highest cotinine strata. The difference between controls and asthmatics remained significant for B-Eos in all cotinine strata (table 4). The direction of the effect of increasing cotinine levels on the B-Eos count was different in controls and asthmatics, with fewer controls than asthmatics showing a reduction in B-Eos compared with the reference stratum (table E1).

The effect of increasing cotinine on FeNO and B-Eos was consistent when dividing the participants by gender (tables E2 and E3). In addition, there was no significant effect of recent smoking, as defined in the question.

A similar effect of tobacco smoke exposure on FeNO and B-Eos was also seen in non-smoking controls (never smokers plus former smokers), whereas passive smoke exposure did not seem to affect FeNO in asthmatics (figure 3).

When looking specifically at participants with cotinine levels $<0.015 \mathrm{ng} \mathrm{ml}^{-1}$, there was a significant difference in FeNO between never smokers and 
Table 3. Effect of increasing serum cotinine on FeNO in the control and asthma groups.

\begin{tabular}{|c|c|c|c|c|c|}
\hline \multirow[b]{2}{*}{ Cot. $\left(\mathrm{ng} \mathrm{ml} \mathrm{m}^{-1}\right)^{*}$} & \multicolumn{2}{|c|}{ Controls } & \multicolumn{2}{|r|}{ Asthma } & \multirow[b]{2}{*}{$p$} \\
\hline & $n$ & FeNO & $n$ & FeNO & \\
\hline \multicolumn{6}{|l|}{ All individuals } \\
\hline$\leqslant 0.015$ & 2453 & $13.2(12.9-13.5)$ & 157 & $19.8(17.5-22.4)$ & $<0.001$ \\
\hline $0.016-0.021$ & 867 & $13.2(12.6-13.8)$ & 62 & $19.4(15.5-24.2)$ & $<0.001$ \\
\hline $0.022-0.122$ & 2935 & $13(12.7-13.3)$ & 235 & $17.7(15.9-19.8)$ & $<0.001$ \\
\hline $0.123-239.6$ & 2903 & $10.9(10.6-11.1)$ & 282 & $14.8(13.3-16.5)$ & $<0.001$ \\
\hline$>239.6$ & 711 & $7.6(7.3-7.9)$ & 64 & $7.4(6.2-8.7)$ & 0.726 \\
\hline \multicolumn{6}{|l|}{ Age $<18$ years } \\
\hline$\leqslant 0.015$ & 677 & $10.3(9.8-10.8)$ & 65 & $18.4(14.7-23)$ & $<0.001$ \\
\hline $0.016-0.020$ & 206 & $10.3(9.4-11.2)$ & 15 & $20.4(10.6-39.4)$ & $<0.001$ \\
\hline $0.021-0.078$ & 740 & $10.1(9.7-10.6)$ & 90 & $17.7(14.3-21.8)$ & $<0.001$ \\
\hline $0.079-2.315$ & 701 & $9.8(9.4-10.3)$ & 115 & $14.7(12.3-17.5)$ & $<0.001$ \\
\hline$>2.315$ & 183 & $9.2(8.4-10.1)$ & 21 & $14.1(8.6-23.2)$ & 0.009 \\
\hline \multicolumn{6}{|l|}{ Age $\geqslant 18$ years } \\
\hline$\leqslant 0.015$ & 1776 & $14.5(14.1-14.8)$ & 92 & $20.8(18-24)$ & $<0.001$ \\
\hline $0.016-0.021(1.6)$ & 614 & $14.7(14-15.4)$ & 41 & $18(14.2-22.8)$ & $<0.001$ \\
\hline $0.022-0.164(2.3)$ & 2245 & $14.3(14-14.7)$ & 143 & $18.3(16.1-20.8)$ & $<0.001$ \\
\hline $0.165-283.0(6.5)$ & 2202 & $10.8(10.5-11.1)$ & 174 & $13.7(12-15.8)$ & $<0.001$ \\
\hline$>283.0(12.7)$ & 525 & $7.5(7.1-7.9)$ & 44 & $7.6(6.2-9.2)$ & 0.941 \\
\hline
\end{tabular}

First category ' $\leqslant 0.015$ ' represents detection limit and is the reference category. FeNO presented as geometric mean (95\% CI). Categories represent percentiles 10, 50, and 90. ${ }^{*}$ Cigarettes smoked per day represented by geometric mean (in brackets). The $p$ value reports the statistical significant difference between Controls and Asthma in each cotinine stratum. Numbers shown in brackets represent the geometric mean of the self-reported average number of cigarettes per day in each cotinine stratum. Please note the different cotinine strata in children and adults.

former smokers in controls and asthmatics $(p=0.001$ and $p<0.001$, respectively), but was not consistent after adjusting for age and gender $(p=0.47$ and $p=0.31$, respectively). However, never smoking controls had significantly lower B-Eos count than former smokers, even after adjusting for age and gender $(p<$ 0.001) (figure 4).

Furthermore, there was no significant effect of recent cigarette smoking on FeNO, defined as smoking in the hour prior to FeNO measurement, in selfreported smokers, regardless of asthma diagnosis and gender, after adjustment for age and serum cotinine (table E4).

\section{Discussion}

This study is the first to report the effect of objectively measured exposure to cigarette smoke both on exhaled $\mathrm{NO}$ and blood eosinophils. The effects were compared in healthy controls and asthmatics, with children and adults analysed separately. Increasing serum cotinine levels were associated with lower FeNO, starting at cotinine levels corresponding to approximately six cigarettes per day in active smokers; this effect appeared to be slightly larger in asthmatics than controls. However, FeNO remained higher in asthmatics than controls for all cotinine strata except the highest decile, corresponding to an average consumption of 13 cigarettes per day. The effect of increasing cotinine levels on B-Eos count was different, with a significant increase in controls and non-significant variations in asthmatics. Interestingly, past active cigarette smoking seemed to be related to persistent type- 2 inflammation, as indicated by increased B-Eos count in presently non-exposed former smokers compared with never smokers.

Cigarette smoking has long been known to reduce FeNO in healthy subjects [21]. This effect was recently studied using the NHANES dataset, but with children and adults grouped together [22]. However, cigarette smoke exposure differs greatly between children and adults (present data) and normal FeNO is lower in children than adults [20], which strongly suggests that children and adults should be analysed separately. Further, Xu et al [22] did not report the effect on FeNO in terms of absolute values and did not relate cotinine levels to cigarette smoke consumption, which makes our study more useful for clinicians. Exhaled NO is also reduced in smokers with asthma, but FeNO may still be used to differentiate asthmatic from nonasthmatic subjects [23]. The relative effect of smoking on FeNO in subjects with asthma versus controls has not been examined previously in a large population. Further, the effect of smoke exposure on FeNO and 
Table 4. Effect of increasing serum cotinine on B-Eos in the control and asthma groups.

\begin{tabular}{|c|c|c|c|c|c|}
\hline \multirow[b]{2}{*}{ Cot. $\left(n g \mathrm{ml}^{-1}\right)^{*}$} & \multicolumn{2}{|c|}{ Controls } & \multicolumn{2}{|c|}{ Asthma } & \multirow[b]{2}{*}{$p$} \\
\hline & $n$ & B-Eos & $n$ & B-Eos & \\
\hline \multicolumn{6}{|l|}{ All individuals } \\
\hline$\leqslant 0.015$ & 2453 & $122(119-126)$ & 157 & $212(185-242)$ & $<0.001$ \\
\hline $0.016-0.021$ & 867 & $128(122-135)$ & 62 & $226(181-282)$ & $<0.001$ \\
\hline $0.022-0.122$ & 2935 & $122(118-125)$ & 235 & $208(185-234)$ & $<0.001$ \\
\hline $0.123-239.6$ & 2903 & $134(131-138)$ & 282 & $213(192-236)$ & $<0.001$ \\
\hline$>239.6$ & 711 & $141(134-149)$ & 64 & $212(178-252)$ & $<0.001$ \\
\hline \multicolumn{6}{|l|}{ Age $<18$ years } \\
\hline$\leqslant 0.015$ & 677 & $125(118-132)$ & 65 & $268(215-334)$ & $<0.001$ \\
\hline $0.016-0.020$ & 206 & $128(116-141)$ & 15 & $302(197-464)$ & $<0.001$ \\
\hline $0.021-0.078$ & 740 & $127(121-134)$ & 90 & $253(207-308)$ & $<0.001$ \\
\hline $0.079-2.315$ & 701 & $136(129-143)$ & 115 & $259(217-310)$ & $<0.001$ \\
\hline$>2.315$ & 183 & $137(122-153)$ & 21 & $214(165-278)$ & 0.007 \\
\hline \multicolumn{6}{|l|}{ Age $\geqslant 18$ years } \\
\hline$\leqslant 0.015$ & 1776 & $121(117-126)$ & 92 & $179(152-210)$ & $<0.001$ \\
\hline $0.016-0.021(1.6)$ & 614 & $129(122-137)$ & 41 & $197(148-262)$ & $<0.001$ \\
\hline $0.022-0.164(2.3)$ & 2245 & $120(116-124)$ & 143 & $181(157-209)$ & $<0.001$ \\
\hline $0.165-283.0(6.5)$ & 2202 & $136(132-140)$ & 174 & $188(167-213)$ & $<0.001$ \\
\hline$>283.0(12.7)$ & 525 & $133(124-142)$ & 44 & $225(187-270)$ & $<0.001$ \\
\hline
\end{tabular}

First category ' $\leqslant 0.015$ ' represents detection limit and is the reference category. FeNO presented as geometric mean (95\% CI). Categories represent percentiles 10, 50, and $90 .{ }^{*}$ Cigarettes smoked per day represented by geometric mean (in brackets). The $p$ value reports the statistical significant difference between Controls and Asthma in each cotinine stratum. Numbers shown in brackets represent the geometric mean of the self-reported average number of cigarettes per day in each cotinine stratum. Please note the different cotinine strata in children and adults.

B-Eos count has not previously been studied in the same subjects.

There are multiple mechanisms proposed to explain the effect of cigarette smoke exposure on FeNO. It has been evidenced by a series of studies that $\mathrm{NO}$ in orally exhaled air primarily originates in the respiratory epithelium, produced by inducible $\mathrm{NO}$ synthase (iNOS) [4]. The basal expression of iNOS in the human respiratory epithelium has been suggested to be regulated by interferon gamma (IFN- $\gamma$ ), which is present in normal airways [24, 25]. Further, several studies have shown lower levels of IFN- $\gamma$ and IFN- $\gamma$ expressing cells in the airways of smokers than in those of non-smokers [15, 26, 27]. The effect of smoking on the formation of IFN- $\gamma$ in the airways may be mediated by acrolein and similar vapour phase compounds acting on T-cells [26]. Another possible mechanism is the reduction of L-arginine bioavailability in the mucosa through the upregulation of epithelial arginase-1 [28]. However, this seems to lead to L-arginine deficiency in asthmatics only (with higher substrate demand) and not in controls [29], which could explain the finding that the effect on FeNO of increasing cotinine levels was slightly larger in asthmatics than in controls. Further, oxidative processes in the airway mucosa induced by cigarette smoke exposure may lead to the direct consumption or scavenging of $\mathrm{NO}$ [30]. Finally, exhaled NO also originates in the pharyngo-oral tract via non-enzymatic formation from nitrite [4], but this pathway does not seem to be affected by smoking [31].

The association of the increase in B-Eos in healthy controls with increasing ongoing cigarette smoke exposure confirms previous observations [31-34]. Taylor et al [33] also reported increased levels of serum total IgE in smokers, a phenomenon that has been shown by others [35]. The increase of serum IgE in smokers, although not available in the present dataset, has been shown to be similar to that in atopic individuals [36]. However, the B-Eos count was not significantly affected by cigarette smoke exposure in asthmatic subjects. The mechanism behind the increased B-Eos count in controls may involve epithelial damage and the expression of alarmins, for example thymic stromal lymphopoietin (TSLP), which have the potential to augment type- 2 inflammation [37] and IgE formation. In asthma, TSLP is already upregulated [38] and, therefore, cigarette smoke exposure may add little to TSLP expression, explaining the lack of effect on B-Eos count in asthmatics.

A reduction in FeNO and an increase in B-Eos count was also seen in controls following second-hand smoke exposure in this study, whereas FeNO was not reduced in asthmatics following second-hand exposure (there was even a trend toward an increase). This is highly relevant given the current knowledge of the 


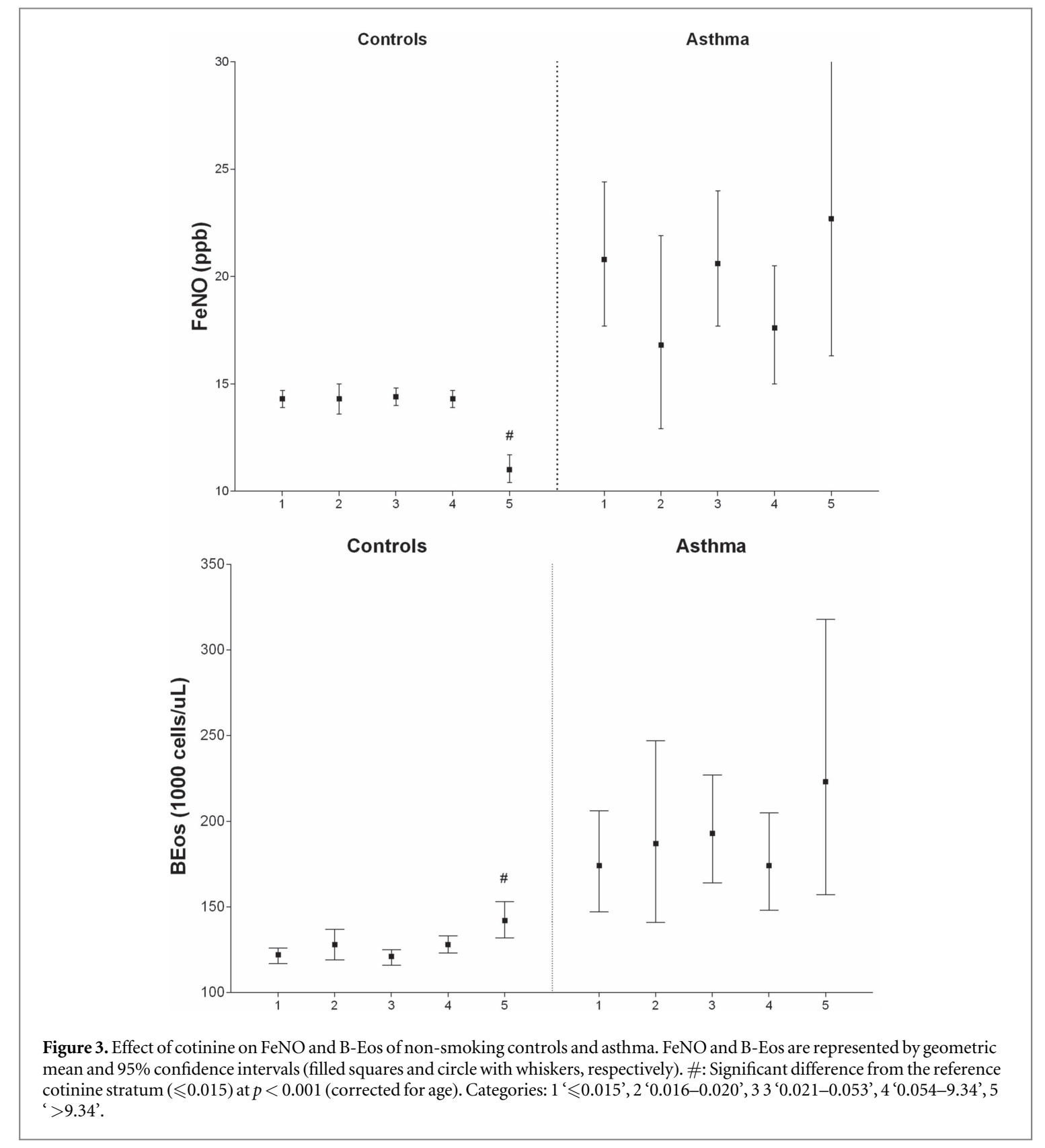

negative effect of second-hand exposure to cigarette smoke, with the most relevant being the depressive effect on IFN- $\gamma$, but no change, or even an increase, in type- 2 cytokines and serum IgE [39, 40].

FeNO has previously been reported to return to expected levels within a few weeks after smoking cessation [41, 42]. However, only a few subjects reported having quit smoking within the past few weeks in the NHANES material, making it difficult to study the the time course of the normalization of FeNO. Our results showed no significant difference in FeNO between former and never smokers, with the analysis undertaken only in subjects not presently exposed to cigarette smoke (undetectable serum cotinine) for the first time. In contrast, this does not seem to be the case for blood eosinophils, which showed persistently elevated levels in former smokers without asthma. Similarly, the sputum eosinophil count was shown not to change, whereas FeNO was normalized, after smoking cessation in young asthmatics [42]. This effect may be mediated by persistent TSLP formation in the airways of former smokers [43].

Recent smoke exposure has previously been suggested to be related to further reduced FeNO [22]. However, cigarette smoking has been shown to cause both an acute reduction [44] and an increase [45] in FeNO, and the relation between recent cigarette smoking (within $1 \mathrm{~h}$ ) and reduced FeNO disappeared after correcting for serum cotinine levels in our material. Since cotinine has a half-life of 15-20 h in blood [18], this indicates that recent smoke exposure is a proxy for daily cigarette smoke consumption, whereas the actual acute effect of cigarette smoke exposure is small and variable.

The interpretation of the results of this study should take into consideration its strengths and 

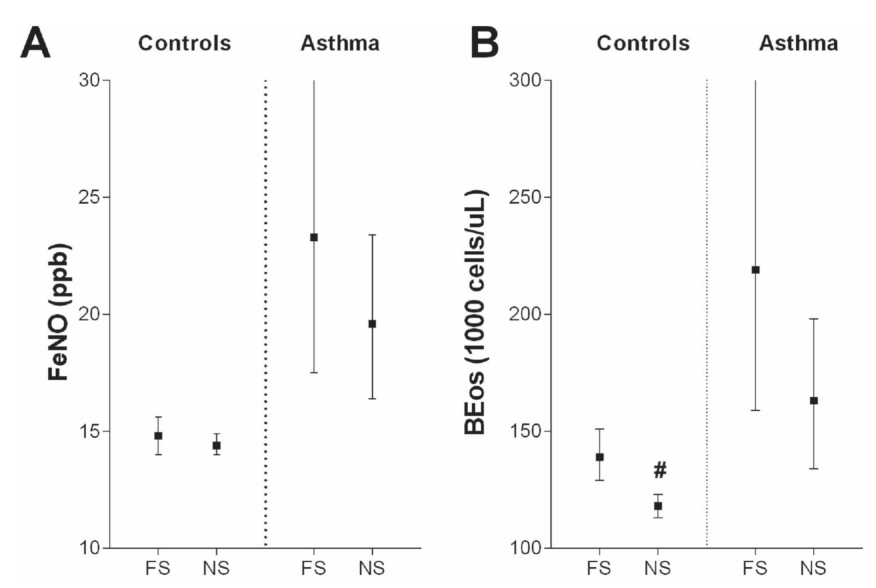

Figure 4. FeNO and B-Eos in never and former smokers with cotinine levels below the LLD, in controls and asthma. FeNO and B-Eos are represented by geometric mean and $95 \%$ confidence intervals (filled squares and circle with whiskers, respectively). \#: Significant difference at $p<0.001$ (corrected for age and gender). Panel A: FeNO; Panel B: B-Eos. FS: former smokers, NS: never smokers.

limitations. The study has a large population-based sample, retrieved from datasets of an ongoing, wellknown and standardized national survey, with highly controlled standard operating procedures and data quality controls. Both FeNO and B-Eos values were from standardized, valid and reproducible measurements. In spite of the difficulty of an accurate determination of cigarette smoke exposure [13, 46], the majority of studies have used self-reported measures for smoking status and second-hand exposure. In this study, we have used serum cotinine, an objective marker that increases the reliability of the analysis, in particular for the detection of passive exposure in never and former smokers. However, the assessment of exposure to cigarette smoke using cotinine, although more adequately measured in blood than in, for example, saliva [14], may have some inherent flaws that could hamper the interpretation-inter-subject variance in nicotine metabolism, time of day of sample collection, and lack of adjustment of cigarette nicotine content [47]. The classification of respiratory diseases and the use of inhaled and oral medication was based on self-reported data, but efforts were made to utilize the best possible combination of questions for the definition of asthma [48].

To conclude, in a large population-based sample of healthy individuals and individuals with self-reported asthma, we showed a negative association of objectively determined exposure to cigarette smoke with exhaled nitric oxide levels and a positive association with blood eosinophil counts. Similar associations were also seen for second-hand smoke exposure. These effects should be taken into consideration in the development of normalized values for these biomarkers [20,22] and in their interpretation in clinical practice. Furthermore, active cigarette smoking seems to elicit type-2 inflammation that persists after smoking cessation. The persistence of elevated blood eosinophils in former smokers should be studied prospectively in order to understand the risk of future inflammatory disease.

\section{Acknowledgments}

This study was supported by the Swedish Foundation for Strategic Research (RB13-0196), the Swedish Heart and Lung Foundation (20160618), and the Swedish Asthma and Allergy Association's Research Foundation (F2016-0045).

\section{Authors contribution}

TJ and KA contributed to study design, data analysis and interpretation, and writing. JAF contributed to study design, data interpretation and writing. AM and CJ contributed to data interpretation and writing. 


\section{References}

[1] 2015 Global Initiative for Asthma. Global Strategy for Asthma Management and Prevention, 2015 (http://ginasthma.org/ local/uploads/files/GINA_Report_2015_Aug11.pdf)

[2] Froidure A et al 2016 Asthma phenotypes and IgE responses Eur. Respir. J. 47 304-19

[3] Malinovschi A et al 2013 Exhaled nitric oxide levels and blood eosinophil counts independently associate with wheeze and asthma events in National Health and Nutrition Examination Survey subjects J. Allergy. Clin. Immunol. 132 821-7

[4] Alving K and Malinovschi A 2010 Basic aspects of exhaled nitric oxide Exhaled Biomarkers 49 (Lausanne: European Respiratory Society) pp 1-31

[5] Dweik R A et al 2011 An official ATS clinical practice guideline: interpretation of exhaled nitric oxide levels (FeNO) for clinical applications Am. J. Respir. Crit. Care Med. 184602-15

[6] Burrows B et al 1980 Epidemiologic observations on eosinophilia and its relation to respiratory disorders 1-3 Am. Rev. Respir. Dis. 122 709-19

[7] Malinovschi A et al 2016 Simultaneously increased fraction of exhaled nitric oxide levels and blood eosinophil counts relate to increased asthma morbidity J. Allergy Clin. Immunol. 138 1301-8

[8] Jacinto T et al 2013 Setting reference values for exhaled nitric oxide: a systematic review Clin. Respir. J. 7 113-20

[9] Kauffmann F et al 1986 Eosinophils, smoking, and lung function. An epidemiologic survey among 912 working men Am. Rev. Respir. Dis. $1341172-5$

[10] Malinovschi A et al 2006 Effect of smoking on exhaled nitric oxide and flow-independent nitric oxide exchange parameters Eur. Respir. J. 28 339-45

[11] Dippolito Retal 2001 Eosinophils in induced sputum from asymptomatic smokers with normal lung function Respir. Med. 95 969-74

[12] Spencer K and Cowans N J 2013 Accuracy of self-reported smoking status in first trimester aneuploidy screening Prenat. Diagn. 33 245-50

[13] Ebner N et al 2013 Assessment of serum cotinine in patients with chronic heart failure: self-reported versus objective smoking behaviour Clin. Res. Cardiol. 102 95-101

[14] Sepkovic D W and Haley N J 1985 Biomedical applications of cotinine quantitation in smoking related research Am. J. Public Health 75 663-5

[15] Tsoumakidou M et al 2007 Cigarette smoking alters bronchial mucosal immunity in asthma Am. J. Respir. Crit. Care Med. 175 919-25

[16] Ho Get al 2013 Biomarkers of tobacco smoke exposure and asthma severity in adults Am. J. Prev. Med. 45 703-9

[17] American Thoracic Societyand European Respiratory Society 2005 ATS/ERS recommendations for standardized procedures for the online and offline measurement of exhaled lower respiratory nitric oxide and nasal nitric oxide, $2005 \mathrm{Am}$. J. Respir. Crit. Care Med. 171 912-30

[18] Jarvis M J et al 1988 Elimination of cotinine from body fluids: implications for noninvasive measurement of tobacco smoke exposure Am. J. Public Health 78 696-8

[19] Benowitz N L 1999 Biomarkers of environmental tobacco smoke exposure Environ. Health Perspect. $107349-55$

[20] Jacinto T et al 2015 Evolution of exhaled nitric oxide levels throughout development and aging of healthy humans J. Breath Res. 936005

[21] Persson M G et al 1994 Single-breath nitric oxide measurements in asthmatic patients and smokers Lancet 343 146-7

[22] Xu X et al 2016 A population-based study of smoking, serum cotinine and exhaled nitric oxide among asthmatics and a healthy population in the USA Inhal. Toxicol. 28 724-30

[23] Malinovschi A et al 2012 The value of exhaled nitric oxide to identify asthma in smoking patients with asthma-like symptoms Respir. Med. 106 794-801
[24] Guo F H et al 2000 Molecular mechanisms of increased nitric oxide (NO) in asthma: evidence for transcriptional and posttranslational regulation of NO synthesis J. Immunol. 164 5970-80

[25] Guo F H et al 1997 Interferon gamma and interleukin 4 stimulate prolonged expression of inducible nitric oxide synthase in human airway epithelium through synthesis of soluble mediators J. Clin. Invest. 100 829-38

[26] Lambert C et al 2005 Acrolein in cigarette smoke inhibits T-cell responses J. Allergy Clin. Immunol. 116 916-22

[27] Meuronen A et al 2008 Decreased cytokine and chemokine mRNA expression in bronchoalveolar lavage in asymptomatic smoking subjects Respiration 75 450-8

[28] Bergeron C et al 2007 Influence of cigarette smoke on the arginine pathway in asthmatic airways: increased expression of arginase I J. Allergy Clin. Immunol. 119391-7

[29] Bruce C T et al 2010 1-Arginine reverses cigarette-induced reduction of fractional exhaled nitric oxide in asthmatic smokers Inflammopharmacology 18 9-16

[30] Ricciardolo F L M et al 2004 Nitric oxide in health and disease of the respiratory system Physiol. Rev. 84 731-65

[31] Marteus $\mathrm{H}$ et al 2004 Nitric oxide formation in the oropharyngeal tract: possible influence of cigarette smoking Nitric Oxide 11 247-55

[32] Lewis S A et al 2001 The relation between peripheral blood leukocyte counts and respiratory symptoms, atopy, lung function, and airway responsiveness in adults Chest 119 105-14

[33] Taylor R Get al 1985 Smoking, allergy, and the differential white blood cell count Thorax 40 17-22

[34] Schwartz J and Weiss S T 1994 Cigarette smoking and peripheral blood leukocyte differentials Ann. Epidemiol. 4 236-42

[35] Burrows B et al 1981 The relationship of serum immunoglobulin E to cigarette smoking Am. Rev. Respir. Dis. 124523-5

[36] Sherrill D L, Halonen M and Burrows B 1994 Relationships between total serum IgE, atopy, and smoking: a twenty-year follow-up analysis J. Allergy Clin. Immunol. 94 954-62

[37] Segawa R and Hirasawa N 2014 Exacerbation of allergic diseases by chemicals: role of TSLP J. Pharmacol. Sci. 124301-6

[38] Gauvreau GM et al 2014 Effects of an anti-TSLP antibody on allergen-induced asthmatic responses N. Engl. J. Med. 370 2102-10

[39] Tebow G et al 2008 Effects of parental smoking on interferon production in children Pediatrics 121 e1563-9

[40] Feleszko W et al 2006 Parental tobacco smoking is associated with augmented IL-13 secretion in children with allergic asthma J. Allergy Clin. Immunol. 117 97-102

[41] Högman M et al 2002 Increased nitric oxide elimination from the airways after smoking cessation Clin. Sci. 103 15-9

[42] Westergaard C G, Porsbjerg C and Backer V 2014 The effect of smoking cessation on airway inflammation in young asthma patients Clin. Exp. Allergy 44 353-61

[43] Nagasaki T et al 2013 Smoking attenuates the age-related decrease in IgE levels and maintains eosinophilic inflammation Clin. Exp. Allergy 43 608-15

[44] Kougias M et al 2013 The acute effect of cigarette smoking on the respiratory function and FENO production among young smokers Exp. Lung Res. 39 359-64

[45] Chambers D C, Tunnicliffe W S and Ayres J G 1998 Acute inhalation of cigarette smoke increases lower respiratory tract nitric oxide concentrations Thorax 53 677-9

[46] Hawkins S S et al 2014 Secondhand smoke exposure among nonsmoking pregnant women in New York City Nicotine Tobacco Res. 16 1079-84

[47] Wall M A et al 1988 Cotinine in the serum, saliva, and urine of nonsmokers, passive smokers, and active smokers Am. J. Public Health 78 699-701

[48] Sá-Sousa A et al 2014 Operational definitions of asthma in recent epidemiological studies are inconsistent Clin. Transl. Allergy 424 